\title{
Evaluation of a New Formula for Calculating Heparin Dose for Cardiopulmonary Bypass Patients
}

\author{
Aya Nakasuji ${ }^{1}$, Satoshi Matsushita ${ }^{1}$, Hirotaka Inaba ${ }^{1}$, Taira Yamamoto ${ }^{1}$, Kenji Kuwaki ${ }^{1}$, \\ Eiichi Inada², Atsushi Amano' \\ ${ }^{1}$ Department of Cardiovascular Surgery, Faculty of Medicine, Juntendo University, Tokyo, Japan \\ ${ }^{2}$ Department of Anesthesiology and Pain Medicine, Faculty of Medicine, Juntendo University, Tokyo, Japan \\ Email: *saty-m@juntendo.ac.jp
}

Received 17 March 2014; revised 17 April 2014; accepted 25 April 2014

Copyright (C) 2014 by authors and Scientific Research Publishing Inc.

This work is licensed under the Creative Commons Attribution International License (CC BY).

http://creativecommons.org/licenses/by/4.0/

c) (i) Open Access

\begin{abstract}
Introduction: Anticoagulation is essential during CPB in cardiac surgery. It is generally performed using heparin; however the widely used formula for its dosing is based solely on body weight. Although the formula assumes activated clotting time (ACT) to be within normal range, baseline ACT varies in each patient. Thus, we developed an original formula, which takes into account baseline ACT in addition to body weight to calculate a more proper dose for initial administration of heparin. In this study, we monitored the ACT to examine if the dose of heparin calculated using our formula can prolong the ACT to the target range, and we determined the factors which interfere with the prolongation of ACT. Methods: Between October 2010 and April 2011, 141 consecutive patients underwent cardiac surgery requiring cardiopulmonary bypass at our hospital. We measured ACT 3 minutes after the initial administration of heparin and considered ACT values $>400$ seconds as appropriate for safe initiation of CPB. Results: Using the proposed formula, administered heparin dose was $241 \pm 27 \mathrm{IU} / \mathrm{kg}$ and target ACT was achieved in $86.4 \%$ of patients. Multivariate analysis was performed to determine the effect of patient background factors on target ACT achievement. Body weight, age, and preoperative heparin therapy, which showed significant differences, were further analyzed. Conclusions: This study demonstrated that our newly developed formula could be used to properly calculate the optimal initial dose of heparin.
\end{abstract}

\section{Keywords}

Anesthesia, Cardiac Surgery, Cardio Pulmonary Bypass

\footnotetext{
${ }^{*}$ Corresponding author.
} 


\section{Introduction}

Since cardiopulmonary bypass (CPB) was introduced for cardiac surgery in 1953, this technological advance has been contributing to the safety of cardiac procedures [1]. During CPB, coagulation control is indispensable. Except in case of allergy, heparin is universally used for anticoagulation to prevent thromboembolism in the CPB circuit. Activated clotting time (ACT) is used to monitor the effect of heparin [1]. If the initial dose of heparin is insufficient, additional heparin is required to reach the minimum ACT value. Currently, 300 IU/kg is widely used as the initial dose of heparin administered before starting CPB [2] [3]. Calculation of this value is solely dependent on patient's body weight (BW), assuming that the ACT value was within normal range. In a retrospective analysis of our data, however, the baseline ACT was $>100$ seconds in most cases. In fact, the ACT value after the administration of initial dose of heparin (300 IU/kg) was sometimes beyond our expectation. Heparin overdose has been reported to be associated with significantly increased postoperative blood loss [4] and/or a longer intensive care unit stay [5] [6]. When an overdose of heparin is administered, the ACT value is likely to increase again after surgery [7]-[9]. Although serum heparin is neutralized by a proper dose of protamine after termination of CPB, heparin accumulated in the tissue is released back into the blood. Therefore, it is important to minimize the initial dose of heparin to prevent postoperative complications. Recently, we developed an original formula that can calculate a more accurate initial dose of heparin based on the ACT value. The purpose of this study was to verify that the dose of heparin calculated by our formula is enough to reach the target ACT for CPB. Further, we determine which factor(s) affect heparin activity by comparing patients who reached the target ACT range after initial administration of heparin with those who did not.

\section{Materials and Methods}

According to the conventional formula, a dose of $300 \mathrm{IU} / \mathrm{kg}$ heparin is administered to reach the target ACT for safe use of cardiopulmonary bypass (CPB). The formula we propose takes into account not only body weight (BW) but also the baseline ACT (bACT) of each patient, while the conventional formula assumes a bACT of 100 seconds for all patients;

$$
\text { Initial dose of heparin }(\mathrm{IU})=\mathrm{BW}(\mathrm{kg}) * 300 *(450-\mathrm{bACT}) /(450-100)
$$

The initial dose of heparin was calculated to achieve an ACT of 450 seconds, and CPB was initiated only after ithad exceeded the target value of 400 seconds, hence the formula allows 50 seconds margin for safety.

The dose of heparin was calculated by our formula using the bACT obtained prior to CPB initiation. Each patient received the calculated dose of heparin through a central venous catheter before aortic cannulation, and the ACT was measured 3 minutes later to determine the effect of heparin. If the target ACT was not achieved after the first dose, additional heparin was administered. ACT was measured in duplicate using a Hemochron ${ }^{\circledR}$ Response system 3 min after each heparin administration. In addition, all components of the CPB circuit were coated with covalently bonded heparin.

\subsection{Patients}

A total of 141 consecutive adult patients undergoing cardiac surgery with CPB at Juntendo Hospital from October 2010 to April 2011 were studied using our new heparin calculation formula. One case with suspected heparin-induced thrombocytopenia prior to surgery was excluded, leaving 140 study patients. Immediately after entering the operating room, anarterial line was inserted in all patients to collect blood samples for baseline ACT, blood count, and renal and liver function determination.

\subsection{Anesthesia}

All patients were anesthetized using fentanyl (5 - $10 \mu \mathrm{g} / \mathrm{kg})$ supplemented with midazolam $(0.1 \mathrm{mg} / \mathrm{kg}+2 \mathrm{mg})$ and rocuronium $(0.6-0.9 \mathrm{mg} / \mathrm{kg})$ for induction and maintained with sevoflurane, propofol, and remifentanil. After CPB termination, heparin was neutralized with protamine sulfate at a dose 1.2 times that of heparin. Background factors such as age, sex, BW, baseline ACT, heparin dose, ACT, liver and renal function, and preoperative heparin therapy were recorded to analyze the relationship between heparin dose and ACT values.

\subsection{Statistical Analysis}

Data were analyzed using Student's $t$ test for comparison between those who reached a safe ACT > 400 seconds 
and those who did not after heparin administration. We used multivariate analysis to evaluate the effect of background factors on target ACT achievement. The statistical software JMP10 (SAS, Tokyo) was used, and significance was set at $p<0.05$.

\section{Results}

The background information of patients is presented in Table 1. Baseline ACT was $>100$ seconds in all patients, with a mean value of $159.1 \pm 25.7$. The heparin dose calculated using the new formula, $241.3 \pm 25.94 \mathrm{IU} / \mathrm{kg}$, was lower than that calculated (300 IU $/ \mathrm{kg}$ ) using the conventional formula $(p<0.01)$. ACT $>400$ seconds was achieved in 102 of 140 patients (86.4\%). In addition, ACT after heparin administration was $>600$ seconds in 21 patients. No thromboembolic events occurred.

We compared the background information of those who achieved ACT $>400$ seconds with those who did not to investigate factors that could affect target ACT achievement (Table 2). Significant differences were observed in age, BW, hemoglobin, and preoperative heparin therapy. Patients in the group that did not achieve the target ACT were older ( $73.7 \pm 8.4$ vs. $63.9 \pm 14.8, p<0.01)$, lighter ( $50.5 \pm 10.0 \mathrm{~kg}$ vs. $59.3 \pm 13.3 \mathrm{~kg}, p<0.01$ ), and had lower hemoglobin values $(11.6 \pm 1.7 \mathrm{~g} / \mathrm{dl}$ vs. $12.7 \pm 2.06 \mathrm{~g} / \mathrm{dl}, p<0.01)$ than those who achieved the target ACT. A total of 19 patients did not achieve ACT of 400 seconds, 13 of which were preoperatively administered heparin. Multivariate analysis was used to explore background factors that might affect the ACT value. In addition to BW, which is the only factor considered in the conventional formula, age and preoperative heparin therapy were also analyzed.

\subsection{Age}

We classified study subjects into four age groups to compare the rates of target ACT achievement (Figure 1). The achievement rate was $100 \%$ for the youngest group of patients (age $<60$ years), whereas $65 \%$ for patients aged $\geq 80$ years, indicating that the achievement rate decreases with age.

Table 1. Baseline and intraoperative data of patients undergoing cardiopulmonary bypass.

\begin{tabular}{cc}
\hline Patient characteristics $(\mathrm{N}=140)$ & $65.2 \pm 14.4$ \\
Age, years & $71(51 \%)$ \\
Sex, male & $58.1 \pm 13.2$ \\
BW, $\mathrm{kg}$ & $23.6 \pm 3.9$ \\
BMI, $\mathrm{kg} / \mathrm{m}^{2}$ & $6(4.3 \%)$ \\
Chronic kidney disease $\left(\mathrm{eGFR}<30 \mathrm{ml} / \mathrm{min} / 1.73 \mathrm{~m}^{2}\right)$ & $2(1.4 \%)$ \\
\hline
\end{tabular}

Values are shown as mean or number (proportion). BW = Body weight, BMI = Body mass index, eGFR = estimated glomerular filtration rate.

Table 2. Comparison of background data between patients who reached the target ACT and those who did not.

\begin{tabular}{cccc}
\hline & ACT $\geq 400 \mathrm{~s}(\mathrm{~N}=121)$ & ACT $<400 \mathrm{~s}(\mathrm{~N}=19)$ & $p$ value \\
\hline Age, years & $63.9 \pm 14.8$ & $73.7 \pm 8.35$ & $<0.01$ \\
Sex, male & $63(52.1 \%)$ & $8(42.1 \%)$ & 0.42 \\
BW, $\mathrm{kg}$ & $59.3 \pm 13.2$ & $50.5 \pm 10.0$ & $<0.01$ \\
BMI, $\mathrm{kg} / \mathrm{m}^{2}$ & $23.3 \pm 3.9$ & $21.1 \pm 3.1$ & 0.01 \\
Baseline ACT, sec & $158 \pm 26$ & 0.32 & 0.95 \\
Platelets $\geq 3 \times 10^{5} / \mathrm{mm}^{3}$ & $6(5.0 \%)$ & $13(6.0 \%)$ & $<0.01$ \\
Preoperative heparin therapy & $25(20.7 \%)$ & & \\
\hline
\end{tabular}

ACT = activated coagulation time. 


\subsection{Preoperative Heparin Therapy}

We also analyzed preoperative heparin therapy (Table 3).

A total of 38 patients (27.1\%) had received preoperative heparin therapy for $9.9 \pm 7.7$ days. Only 25 of these patients (65.8\%) achieved the target ACT, which was significantly less than 96 of 102 patients $(94.1 \%)$ who did not receive preoperative heparin $(p<0.01)$. Baseline ACT was significantly higher in patients who received preoperative heparin therapy (168.5 \pm 34.6 vs. $153.3 \pm 19.6, p<0.01)$ and their $\mathrm{BW}$ was lower $(53.9 \pm 10.3$ vs. 59.5 $\pm 13.9, p<0.04$ ). We compared variation in ACT with the heparin dose administered to the two groups (Figure 2). The degree of ACT prolongation had significantly less effect in the group that received preoperative heparin $\left(y=1.4935 x, r^{2}=0.0781\right)$ than in the group that did not $\left(y=1.1136 x, r^{2}=0.2591\right)(p<0.05)$.

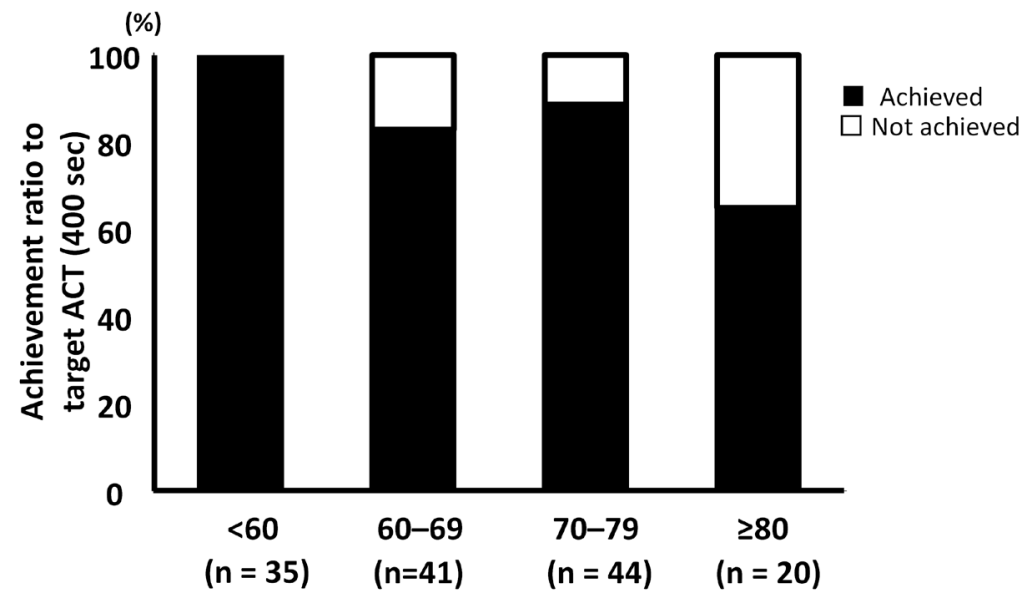

\section{Age (years)}

Figure 1. The achievement ratio to target ACT was compared among the age groups. The youngest group of patients ( $<60$ years) showed an achievement rate of $100 \%$. The rates were $82.9 \%$ in patients aged 60 - 69 years, $88.6 \%$ in those aged 70 - 79 years, and $65 \%$ in patients aged $\geq 80$ years, showing a decrease in the achievement rate with age.

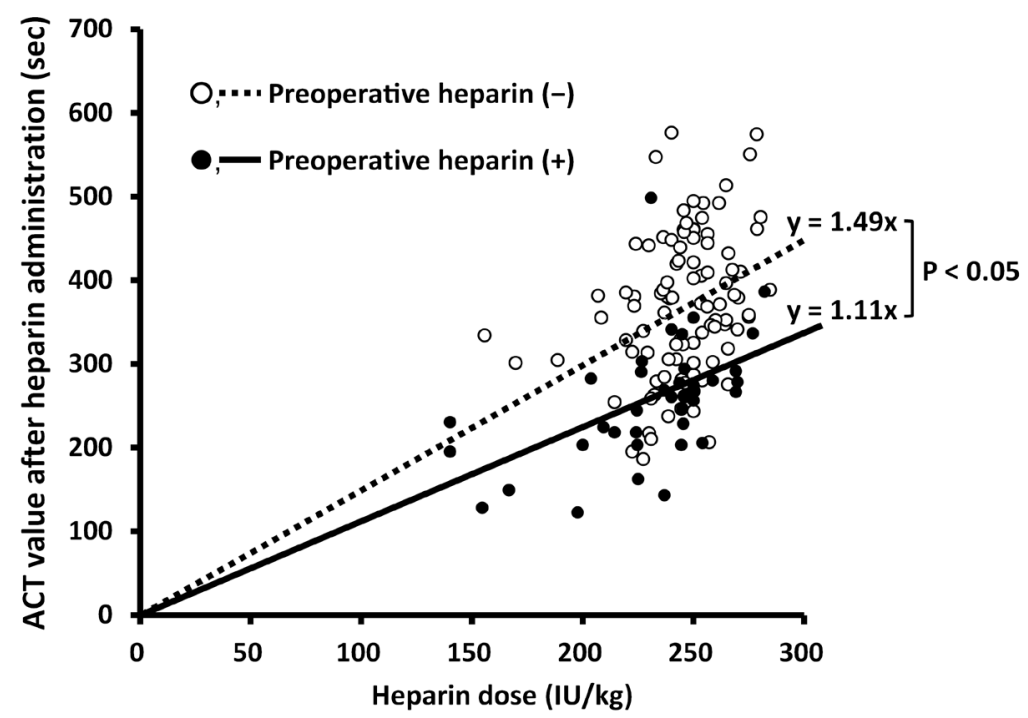

Figure 2. The variation of ACT value in response to the heparin dose was plotted to compare the group that received preoperative heparin and the group that did not. The trendline showed that the response to heparin was significantly lower in the group receiving preoperative heparin $(p<0.05)$. 
Table 3. Comparison of background data between patients with and without preoperative heparin.

\begin{tabular}{cccc}
\hline & Preoperative heparin & No preoperative heparin & $p$ value \\
\hline & $(\mathrm{N}=38)$ & $(\mathrm{N}=102)$ & $<0.08$ \\
\hline Age, years & $68.7 \pm 11.4$ & $63.9 \pm 15.3$ & 0.39 \\
Sex, male & $17(44.7 \%)$ & $54(51.0 \%)$ & 0.04 \\
BW, kg & $54.4 \pm 10$ & $59.5 \pm 14$ & $<0.01$ \\
Baseline ACT, sec & $170 \pm 34$ & $155 \pm 21$ & $<0.01$ \\
Number of reached to target ACT \\
$(\geq 400 \mathrm{sec})$
\end{tabular}

\section{Discussion}

We evaluated whether heparin dose determined using our formula considering baseline ACT in addition to BW was useful and safe for patients undergoing CPB. We found the initial dose of heparin calculated using our formula to be lower than that calculated using the conventional formula, which considers only BW. The rate of target ACT achievement using our formula was $86.4 \%$, which was comparable to Shuhaibar's report of an $82.4 \%$ achievement rate using the conventional dose of $300 \mathrm{IU} / \mathrm{kg}$ heparin [5]. Our results suggest the possibility that the conventional dose of heparin may be excessive and our formula is useful with respect to target ACT achievement. None of our study subjects experienced any thromboembolic events.

Heparin resistance, defined as the failure of $450 \mathrm{IU} / \mathrm{kg}$ heparin to prolong ACT to 480 seconds, has been reported in 10\% - 20\% of cases undergoing CPB [10]-[13]. In this study, there were no cases of heparin resistance; however, additional doses of heparin were required in $15 \%$ of cases despite using the same dose. Ranucci et al. suggested four predictors for heparin resistance: age $\geq 65$ years, platelet count $\geq 300,000$ cells $/ \mathrm{mm}^{3}$, preoperative heparin therapy, and antithrombin III (AT III) levels $\leq 60 \%$ [14]-[16]. Our formula was adjusted for BW after excluding the effect of baseline ACT so that factors affecting the action of heparin would be more definitely extracted. The results suggest that two factors, age and preoperative heparin therapy, should also be considered to administer a proper dose of heparin.

To explore the effect of age on anticoagulation, target ACT achievement was compared among four age groups within the study. Patients aged $<60$ years had $100 \%$ target ACT achievement. On the other hand, 18.1\% of patients in their $60 \mathrm{~s}$ did not achieve the target ACT. The oldest group of patients, age $\geq 80$, had the lowest achievement rate, with $35.0 \%$ of patients not meeting the target ACT. It is commonly said that age is the biggest risk factor for thromboembolism. In the elderly, activation molecular markers of coagulation and fibrinolysis are known to increase. Heparin has the ability to bind to various plasma proteins other than AT III and acts on coagulation factors. AT III and heparin cofactor II undergo a gradual decrease, whereas factor VIII and von Willebrand factor increase [17]. Our results suggest that changes incoagulation and fibrinolysis factors due to age may affect heparin function.

Another factor that affects anticoagulation is preoperative heparin therapy. Approximately $66 \%$ of the patients who received preoperative heparin therapy achieved the target ACT. Heparin resistance occurs because of longterm administration of heparin. The common mechanisms responsible for heparin resistance are increased activity of factor VIII [16] and deficiency of AT III. Heparin shortens the half-life of AT III and decreases the level of circulating AT III; therefore, AT III deficiency is considered the most important factor [16]. Furthermore, because an increase in factor VIII shortens activated partial thromboplastin time regardless of the existence of heparin, this is referred to aspseudo-heparin resistance [18].

Since AT III and factor VIII were not measured in this study, we could not definitively determine the cause of heparin resistance. However, it was suggested that preoperative heparin therapy was responsible for significantly increased heparin resistance.

On the other hand, ACT was $>600$ seconds in 21 patients after heparin administration despite administration of the lower dose calculated by our formula. With regard to age and preoperative heparin therapy, which showed a significant difference, cases with ACT >600 seconds were younger ( $56.9 \pm 19.3$ vs. $66.6 \pm 13.0$ years), and only 1 of 21 patients (4.8\%) had received preoperative heparin therapy, which suggests that we have to reduce heparin dose further in order to prevent excessive administration in cases that have no factors related to heparin 
resistance described previously.

\section{Conclusion}

Recently, we developed a new formula using individual baseline ACT value as well as BW to determine the optimal initial dose of heparin prior to the initiation of CPB. This study demonstrated that the new formula is useful and the dose determined using this formula is safe. Furthermore, age and preoperative heparin therapy, were identified as two additional factors that should be considered when calculating individual heparin dose. By establishing and using a more accurate formula to determine the optimal initial dose at the introduction of CPB, frequent additional dose would fall into disuse, and thus may reduce the risk of postoperative bleeding caused by excessive heparin administration.

\section{Acknowledgements}

We thank Yuko Kojima for correcting the English in this manuscript.

\section{Disclosure Statement}

All authors in this manuscript have no conflict of interest.

\section{References}

[1] Gibbon, J.H. and Passim, J.R. (1954) Application of a Mechanical Heart and Lung Apparatus to Cardiac Surgery. Minnesota Medicine, 37, 171-185.

[2] Bull, B.S., Karpman, R.A., Huse, W.M. and Briggs, B.D. (1975) Heparin Therapy during Extracorporeal Circulation. Problems Inherent in Existing Heparin Protocols. The Journal of Thoracic and Cardiovascular Surgery, 69, 674-684.

[3] Bull, B.S., Huse, W.M., Brauer, F.S. and Korpman, R.A. (1975) Heparin Therapy during Extracorporeal Circulation. II. The Use of a Dose-Response Curve to Individualize Heparin and Protamine Dosage. The Journal of Thoracic and Cardiovascular Surgery, 69, 685-689.

[4] Shuhaibar, M.N., Hargrove, M., Millat, M.H., O’Donnell, A. and Aherne, T. (2004) How Much Heparin Do We Really Need to Go on Pump? A Rethink of Current Practices. European Journal Cardio-Thoracic Surgery, 26, 947-950. http://dx.doi.org/10.1016/j.ejcts.2004.07.009

[5] Fromes, Y., Daghildjian, K., Caumartin, L., Fischer, M., Rouquette, I., Deleuze, P., et al. (2011) A Comparison of Low vs Conventional-Dose Heparin for Minimal Cardiopulmonary Bypass in Coronary Artery Bypass Grafting Surgery. Anaesthesia, 66, 488-492. http://dx.doi.org/10.1111/j.1365-2044.2011.06709.x

[6] Gravlee, G.P., Rogers, A.T., Dudas, L.M.R.N., Taylor, R., et al. (1992) Heparin Management Protocol for Cardiopulmonary Bypass Influences Postoperative Heparin Rebound but Not Bleeding. Anesthesiology, 76, 93-401. http://dx.doi.org/10.1097/00000542-199203000-00012

[7] John, L.C., Rees, G.M. and Kovacs, I.B. (1993) Inhibition of Platelet Function by Heparin. An Etiologic Factor in Post Bypass Hemorrhage. The Journal of Thoracic and Cardiovascular Surgery, 105, 816-822.

[8] Purandare, S.V., Parulkar, G.B., Panday, S.R., Bhattacharya, S. and Bhatt, M.M. (1979) Heparin Rebound a Cause of Bleeding Following Open Heart Surgery. Journal of Postgraduate Medicine, 25, 70-74.

[9] Teoh, K.H.T., Young, E., Blackall, M.H., Roberts, R.S. and Hirsh, J. (2004) Can Extra Protamine Eliminate Heparin Rebound Following Cardiopulmonary Bypass Surgery? The Journal of Thoracic and Cardiovascular Surgery, 128, 211-219. http://dx.doi.org/10.1016/j.jtcvs.2003.12.023

[10] Ravi, T. and Chenq, D.C. (2006) Heparin-Bonded Cardiopulmonary Bypass Circuits Should Be Routine for All Cardiac Surgical Procedures. Anesthesia \& Analgesia, 103, 1370-1372. http://dx.doi.org/10.1213/01.ane.0000223781.83906.1b

[11] Brinks, H.J., Weerwind, P.W., Bogdan, S., Verbruggen, H. and Brouwer, M.H. (2001) Does Heparin Pretreatment Affect the Haemostatic System during and after Cardiopulmonary Bypass? Perfusion, 16, 3-12. http://dx.doi.org/10.1177/026765910101600102

[12] Staples, M.H., Dunton, R.F., Karlson, K.J., Leonardi, H.K. and Berger, R.L. (1994) Heparin Resistance after Preoperative Heparin Therapy or Intraaortic Balloon Pumping. The Annals of Thoracic Surgery, 57, 1211-1216. http://dx.doi.org/10.1016/0003-4975(94)91359-5

[13] Sagripanti, A. and Carpi, A. (1998) Natural Anticoagulants, Aging, and Thromboembolism. Experimental Gerontology, 
33, 891- 896. http://dx.doi.org/10.1016/S0531-5565(98)00047-3

[14] Williams, M.R., D’Ambra, A.B., Beck, J.R., et al. (2000) A Randomized Trial of Antithrombin Concentrate for Treatment of Heparin Resistance. The Annals of Thoracic Surgery, 70, 873-877. http://dx.doi.org/10.1016/S0003-4975(00)01550-2

[15] Ranucci, M., Isgrò, G., Cazzaniga, A., Ditta, A., Boncilli, A., Cotza, M., et al. (2002) Different Patterns of Heparin Resistance: Therapeutic Implications. Perfusion, 17, 199-204. http://dx.doi.org/10.1191/0267659102pf562oa

[16] Ranucci, M., Isgrò, G., Cazzaniga, A., Soro, G., Menicanti, L. and Frigiola, A. (1999) Predictors for Heparin Resistance in Patients Undergoing Coronary Artery Bypass Grafting. Perfusion, 14, 437-442. http://dx.doi.org/10.1177/026765919901400606

[17] Demma, L.J., Winkler, A.M. and Levy, J.H. (2011) FAHA. A Diagnosis of Heparin-Induced Thrombocytopenia with Combined Clinical and Laboratory Methods in Cardiothoracic Surgical Intensive Care Unit Patients. Anesthesia \& Analgesia, 113, 697-702.

[18] O’Donnell, J., Tuddenham, E.G., Manning, R., Kemball-Cook, G., Johnson, D. and Laffan, M. (1997) High Prevalence of Elevated Factor VIII Levels in Patients Referred for Thrombophilia Screening: Role of Increased Synthesis and Relationship to the Acute Phase Reaction. Thrombosis and Haemostasis, 77, 825-828. 